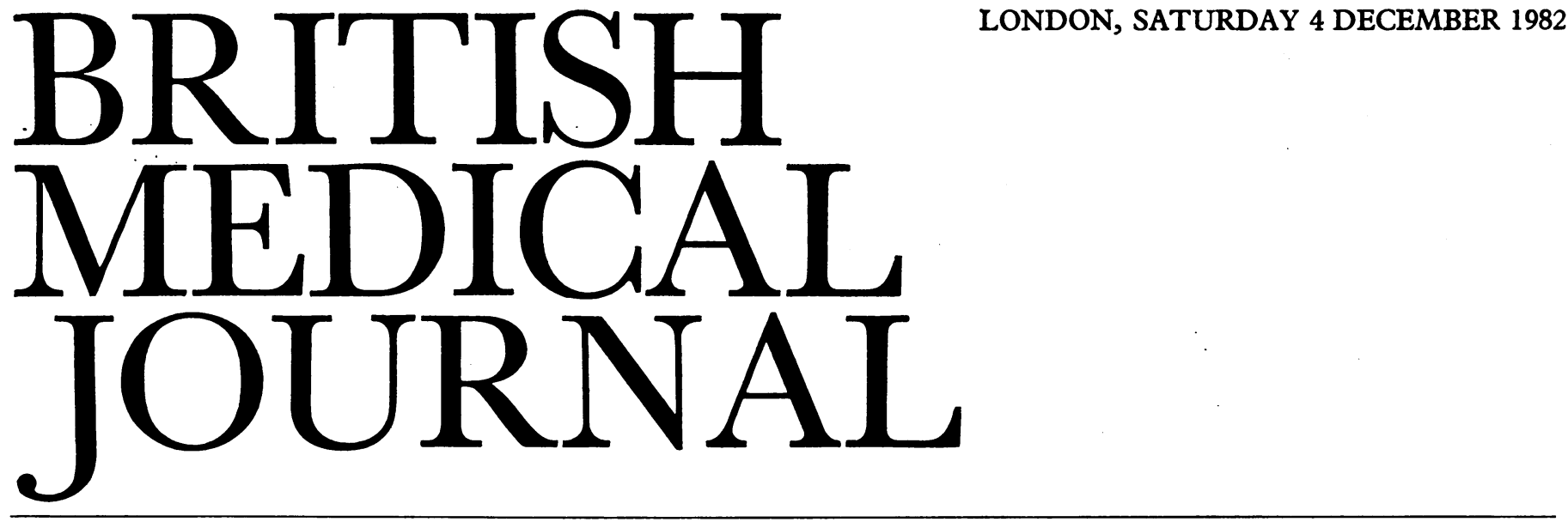

\title{
Can infection be abolished?
}

There is a common but false belief that by now the causes of all bacterial diseases have been discovered. On the contrary new pathogenic bacteria continue to be detected-legionellas, for example, as a cause of pneumonia; and the part played by others is better understood-for example, of Clostridium difficile as a cause of pseudomembranous colitis and of campylobacters as a cause of diarrhoea. New viruses are also appearing, and some that until recently were "orphans in search of a disease" have now found their disease. In the last decade enterovirus 70 has been found in association with a disturbing new disease called acute haemorrhagic conjunctivitis which has swept around the world, particularly the tropics, affecting millions of people and occasionally and exceptionally giving rise to neurological complications. ${ }^{1}$ Africa has recently provided such serious previously unnamed infections as Lassa, Ebola, and Marburg disease. Non-gonococcal urethritis and other sexually transmitted diseases are increasing at an alarming rate-not surprisingly in view of altered patterns of behaviour-and the bacteroides have been reassessed and found to be much more important causes of infections in the chest and abdomen than had been previously supposed.

In the Rock Carling Fellowship lecture for $1982,{ }^{2}$ given at Leicester on 30 November, Dr David Tyrrell asked if the objective of abolishing infection was a hope or an illusion. His answer was that individual infections might disappear from certain areas of the world but infections as a whole could not be abolished: they could only be held in check. Over much of the globe, moreover, they had still to be reduced to a bearably low incidence; there was still much to be done.

Dr Tyrrell's distinguished work at the Medical Research Council's Clinical Research Centre, Northwick Park, and at the Common Cold Unit, Salisbury, has given him a rich harvest of experiences and many international contacts from which to draw his examples and on which to base his examination and discussion of his own question. Through the World Health Organisation and much travel abroad he made many stimulating friends who were also rivals - but not so fiercely competitive as many in other branches of science. They would exchange key reagents and plan studies together when this was worth doing. He was able to work one day a week in medical outpatients at Salisbury General Infirmary at the same time as working on the frontiers of our understanding of viruses. This ensured that he experienced a strong urge to do something about infectious diseases as seen in clinical practice and especially in developing countries, while knowing within his rational self that the most fundamental need was a precise knowledge of infective agents and of the specific means of preventing or treating infections.

More studies of the pathogenesis of infections could be very rewarding. As Tyrrell rightly insists, this area of knowledge calls for studies including microbiology, immunity, and morbid anatomy. Only multidisciplinary studies concerned with unravelling the full range of interactions between host and parasite are likely to yield practical applications of value. In the development of new vaccines and the improvement of old ones such initial studies may show how best to prevent infectionperhaps by inducing antibody directed against the attachment site in the host, or the toxin of the bacterium, or against both. There is hope that an approach on these lines may lead to an improved whooping cough vaccine. The prevention of influenza might also be improved by some elegant studies of its pathogenesis now in progress, and the epidemiology of dengue virus infections might likewise become better understood by similar work. The overall state of the host, including his nutrition, the normal flora of various parts of the body, environment generally, and social and personal behaviour in particular, are all of them relevant in determining the presence or absence of illness due to infective agents. We live surrounded by millions of bacteria and not surprisingly some of them, a minority, sometimes breach our natural defences, especially if these are unwisely neglected or misused.

Our uncritical use of antibiotics often comes from inadequate clinical awareness of how to use these valuable drugs. Each antibiotic has its own possibilities and its own limitations. Not to know and apply what has been learnt about these possibilities and limitations amounts to something pretty close to disgraceful carelessness. Yet it is all too true that these drugs are often marketed, promoted, and used with a deadly indifference to the long term consequences of their misuse-so that we now read with increasing frequency of whole populations of microbes at first fully sensitive to commonly used antibiotics now becoming resistant not to one but to many antibiotics. We need to make better use of antibiotics and combine their use with other approaches-for interrupting the trans- 
mission of resistant organisms by providing and using adequate isolation facilities in hospitals, for example; educate patients not to expect an antibiotic for everything; to ensure that low dose short-period regimens are avoided; and to make determined efforts to persuade the pharmaceutical firms and the Government to get into useful cooperation with each other and with the veterinary and medical professions to prolong the useful life of antibiotics. Failure to build constructively on the recommendations of the Swann Committee on the use of antibiotics in agriculture ${ }^{3}$ may prove in the long term to be as serious an error as it was judged to be in the leading article in the $B M \mathcal{F}$ on the subject. ${ }^{4} \mathrm{Dr}$ Tyrrell rightly says that we are likely to succeed in containing the spread of antibiotic resistant bacteria only if we use all the tactics open to us-there is no one simple and easy way out and no hope of getting away from the problem if we say that no real problem exists and that in due course if we carry on as we are doing all the difficulties will go away.

Valuable new drugs are on the horizon: the antivirals and human interferon, now made more readily available by genetic engineering. It is equally important that we are careful enough in our use of them to ensure that they remain of value for a long time. Genetic engineering should be encouraged with reasonable but not exaggerated safeguards. The prospective dividends are very rich indeed and seem to include the possibility of making safer and more effective synthetic vaccines with a few amino acids instead of a whole microbe. On the general question of laboratory safety the way forward, as Tyrrell wisely sees it, is to resolve differences of opinion and conflicts of interest by relying upon scientific evidence, based on specific and quantitative data wherever possible; by proper consideration of risks in relation to routes of infection; by leaving some matters unsettled pending epidemiological study; and by avoiding attitudes which promote confrontation and mistrust. The problems are real and have to be faced but they must not be exaggerated. The law and common sense demand only what is reasonably practicable and demonstrably necessary.

Organisation and training must be re-examined if we are to develop adequate strategies for the control of infectious diseases. Adequate supplies of pure water and clean food, together with better treatment, more immunisation, better housing, and better isolation facilities, have done much for advanced countries but we may not take these things for granted. The pipework of some sewage collection systems already needs replacement; and the few simple rules of kitchen hygiene necessary for the safe cooking of frozen chickens are often not even dimly understood. Isolation facilities, although better than they were, need to be made still better and be more often and more intelligently used.

Above all, the organisation of our preventive efforts and the training of infectious disease physicians need to be radically upgraded around the general theme of better provision of basic preventive care. There is little drama in preventing infection (indeed often no real evidence to prove success) and certainly no grateful patients. Worst of all there is little esteem from one's fellow doctors. What is required for the best practice and research in infectious diseases is a substantial effort to build up a structure beyond what is needed for the competent practice of a general physician or other specialist-a structure that includes bacteriology, virology, and epidemiology on the one hand and immunology and pathogenesis on the other as well as a detailed understanding of the pharmokinetics, toxicology, and the antimicrobial range of antibiotics. In Tyrrell's view, that means providing an infectious disease unit in a general hospital. There are various directions in which changes would have to be made in existing training and staffing procedures, 3 but clearly academic esteem, at present lacking, should be built up, as it has been in the United States. Staff and students must come to understand that the study of infections is not a matter of past history or present routine but a subject of growing knowledge and interest. He says in plain words that we must not allow academic cuts and lengthy programmes for specialist accreditation to deprive us of that small number of clinician scientists we need and can support. Alas, there is a rumour that a university chair of infectious diseases may remain unfilled after the retirement of its present occupant within the next year. Dr Tyrrell's clear challenge deserves full discussion and an adequate response.

JAMES HowIE

1 Higgins P. Enteroviral conjunctivitis and its neurological complications. Arch Virol (in press).

2 The Rock Carling Fellowship Lecture. The abolition of infection. Hope or illusion? D A J Tyrrell. London: The Nuffield Provincial Hospitals Trust, 1982.

${ }^{3}$ Foint Committee on the Use of Antibiotics in Animal Husbandry and Veterinary Medicine. London: HMSO, 1969, 12.16 and 12.17. (Cmnd 4190; Swann Report.)

4 Anonymous. Death of a quango. $\operatorname{Br} M e d \mathcal{F} 1981$;282:1413-4.

\section{Monoamine oxidase inhibitors in depression}

Depression is a universal experience-and one which in most cases is self-limiting, often psychologically beneficial, and (apart from a little help from our friends) best managed without the intervention of specialists or the use of drugs. Depressive illness or disorder is a very different matter, for here we are dealing with a condition of high morbidity and a substantial mortality-a cause of much suffering and misery to patients and their families.

Unfortunately, depressive illness is not a single entity but a heterogeneous group of disorders and the difficulties are increased by confused terminology. The depressed phase of affective psychoses (ICD, ninth revision), ${ }^{1}$ major affective disorders (American Psychiatric Association criteria), ${ }^{2}$ endogenous depression, and major or primary depressive disorders ${ }^{3}$ all broadly correspond to the same entity characterised by severe depression, retardation or agitation, diurnal variation of $\delta$ mood, and other biological features. These conditions give high $₹$ scores on various depressive indices, and patients usually show 을 a good response to tricyclic antidepressants or electric $N$ convulsion therapy. ${ }^{4}$

Smiling, masked, covert, or hidden depression; atypical facial or other pain; alcohol abuse; and shoplifting or other atypical behaviours are often described as depressive equiva- N lents. Though such terms may help to increase our awareness of the difficulties of diagnosis of primary depressive disorders, 0 there is no justification for treating the disorders described as independent entities. Careful history taking and examination? should result in a proper diagnosis, and soon more help may be available from the laboratory in doubtful cases. ${ }^{5}$

Patients who give low scores on depressive indices and respond poorly to tricyclic antidepressants or electric convulsion therapy present real difficulties and suffer much distress. They are often labelled as having atypical depression, the term used by West and Dally in $1959^{6}$ to describe a group of patients who showed a preferential response to monoamine 